\title{
Competencias del professorado para la atención de la diversidade en el aula
}

\author{
Competences of teachers for the attention of diversity in the classroom
}

Competências dos professores para a atenção da diversidade na sala de aula

\author{
Juan Cornejo-Espejo \\ Profesor doctor de la Universidad del Bío-Bío, Chillán, Chile \\ E-mail: jcornejo@ubiobio.cl ORCID: https://orcid.org/0000-0002-3575-9530
}

Recebido em 30 de agosto de 2021

Aprovado em 20 de novembro de 2021

Publicado em 27 de dezembro de 2021

\section{RESUMEN}

El presente artículo tiene por objetivo analizar la formación que proporcionan las universidades del Consejo de Rectores de las Universidades Chilenas (CRUCH) a los futuros profesores/as, poniendo especial atención a las competencias con que egresan para atender a la diversidad del estudiantado presente en las escuelas nacionales. Diversidad que no se agota sólo en la atención de los/as estudiantes com necesidades educativas especiales (NEE), o en situación de discapacidad, sino que incluye a otros grupos que demandan ser, igualmente, incluidos. Desde el punto de vista metodológico, la investigación se encuadra en un enfoque cualitativo-descriptivo con un diseño de casos múltiples atendiendo a la multiplicidad de unidades de análisis conformadas por las universidades del $\mathrm{CRUCH}$ que imparten pedagogías. Desde el punto de vista de las técnicas de recolección de datos, se privilegió el análisis de documentos provenientes de internet. EI corpus documental estuvo conformado por los itinerarios formativos y los perfiles de egreso declarados en las respectivas páginas webs institucionales. Entre los principales hallazgos, destaca la creciente preocupación de parte de las universidades por la inclusión educativa (IE). No obstante, ella se tiende a concentrar en la preparación de profesores/as para atender a estudiantes con NEE, descuidando tanto a otros grupos, entre ellos indígenas y migrantes, y una perspectiva intercultural, cuanto otras temáticas referidas a género e inclusión de estudiantes disidentes que cuestionan los paradigmas patriarcales y heterosexistas. A modo de conclusión, se establece que el mayor desafío de estas instituciones es ampliar las nociones y grupos beneficiarios de la IE, además de empaparse de la filosofía que subyace a la misma.

Palabras clave: Competencias; Profesores; Inclusión.

\section{ABSTRACT}

The objective of this article is to analyze the training provided by the Council of Rectors of Chilean Universities (CRUCH in Spanish) to future teachers, paying special attention to the skills with which they graduate to attend to the diversity of the students present in 
national schools. Diversity that does not end only in the attention of students with Special Educational Needs (NEE in Spanish), or in a situation of disability, but also includes other groups that claim to be, equally, included. From the methodological point of view, the research is framed in a qualitative-descriptive approach with a multiple-case design attending to the multiplicity of analysis units made up of the $\mathrm{CRCHU}$ universities that teach pedagogies. From the point of view of data collection techniques, the analysis of documents from the Internet was privileged. The documentary corpus was made up of the training itineraries and the graduation profiles declared in the respective institutional web pages. Among the main findings, the growing concern on the part of universities for Educational Inclusion (IE in Spanish) stands out. However, it tends to focus on the preparation of teachers to serve students with SEN, neglecting both other groups, including indigenous and migrants, and an intercultural perspective, as well as other issues related to gender and inclusion of dissident students that question patriarchal and heterosexist paradigms. By way of conclusion, it is established that the greatest challenge for these institutions is to broaden the notions and beneficiary groups of $\mathrm{El}$, as well as to soak up the philosophy that underlies it.

Keywords: Competencies; Teachers; Inclusion.

\section{RESUMO}

O objetivo deste artigo, é analisar a formação proporcionada pelas universidades do Consejo de Rectores de las Universidades Chilenas ( $\mathrm{CRUCH})$ aos futuros professores, dando especial atenção às competências com que se formam para atender à diversidade dos alunos presentes nas escolas nacionais. Diversidade que não se esgota apenas na atenção dos alunos com necesidades educativas especiales (NEE), ou em situação de deficiência, mas considera também outros grupos que exigem ser, igualmente, incluídos. Do ponto de vista metodológico, a pesquisa se enquadra em uma abordagem qualitativodescritiva com desenho de casos múltiplos atendendo à multiplicidade de unidades de análise composta pelas universidades do $\mathrm{CRUCH}$ que ensinam pedagogias. Do ponto de vista das técnicas de coleta de dados, privilegiou-se a análise de documentos da internet. O corpus documental foi constituído pelos itinerários formativos e perfis profissionais declarados nas respetivas páginas webs institucionais. Entre os principais achados, destaca-se a crescente preocupação das universidades com a educação inclusiva (EI). No entanto, ela tende a se concentrar na preparação de professores para atender estudantes com NEE, negligenciando tanto os outros grupos, incluindo indígenas e migrantes, quanto uma perspectiva intercultural, bem como outras questões relacionadas a gênero e inclusão de estudantes dissidentes que questionam os paradigmas patriarcais e heterossexistas. A título de conclusão, estabelece-se que o maior desafio para essas instituições é ampliar as noções e grupos beneficiários da El, além de absorver a filosofia que lhe está subjacente.

Palavras-chave: Competências; Professores; Inclusão. 
http://dx.doi.org/10.5902/1984686X67482

\section{Introducción}

La inclusión educativa (IE) se ha convertido para la formación del profesorado de todos los niveles del sistema escolar chileno (educación preescolar, básica y media). No sólo en una necesidad sino en una exigencia que deviene tanto de los requerimientos del propio sistema escolar y estándares de formación pedagógica (CPEIP, 2016), cuanto de las exigencias emanadas de la Comisión Nacional de Acreditación (CNA, 2019). En esta misma línea, no se pueden obviar también las políticas inclusivas desarrolladas por los distintos gobiernos de turno, ya desde inicios de la década de 1990, y masificadas en las últimas dos décadas, en sintonía con los postulados de la Declaración de Salamanca (1994) y documentos sucesivos de Unesco, que incentivan a los estudiantes con necesidades educativas especiales (NEE) y/o en situación de discapacidad, acceder a las escuelas regulares o comunes, a fin de poner fin o limitar las escuelas segregadas e incentivar la inclusión efectiva de todo el estudiantado, independientemente de sus capacidades cognitivas, físicas $u$ otras peculiaridades.

Atendiendo a esas exigencias y crecientes expectativas sociales, el presente artículo busca dilucidar si la formación que proporcionan las distintas universidades del país formadoras de profesores/as, los/as capacitan, una vez egresados, para atender a la diversidad del estudiantado que frecuentan las escuelas nacionales. Diversidad que no se agota apenas en la atención de los/as estudiantes con NEE o en situación de discapacidad, sino que incluye a otros grupos que demandan ser, igualmente, incluidos como son: estudiantes indígenas o pertenecientes a minorías étnicas, migrantes, disidentes sexuales y/o de género, entre otros. Sin contar con otras especificidades que apuntan a distintos tipos y ritmos de aprendizaje, intereses, sensibilidades, etc.

En este ejercicio evaluativo no se pueden obviar, además, las interseccionalidades de que son portadores esos distintos grupos (de género, de clase, de raza, etc.), que provienen de sus distintas identidades, y que en no pocas oportunidades se superponen, entrecruzan y aún confunden.

Con el fin de dar cumplimiento al objetivo del artículo, se seleccionaron las universidades que hacen parte del Consejo de Rectores de las Universidades Chilenas $(\mathrm{CRUCH})^{1}$ que imparten pedagogías, con exclusión de las universidades privadas ${ }^{2}$. Ello no sólo porque la mayor parte de las universidades del $\mathrm{CRUCH}$ son las más antiguas y prestigiosas del país, sino porque son ellas las que, mayoritariamente, han impulsado las 
http://dx.doi.org/10.5902/1984686X67482

innovaciones curriculares tendientes a incorporar la IE como asignatura(s) en los respectivos itinerarios formativos de las distintas pedagogías.

\section{Desafíos que implica la formación del profesorado en inclusión educativa}

A juicio de Garnique (2012) y Valenzuela, Muñoz y Marfulljensen (2018), gran parte del profesorado que enseña hoy en el sistema escolar, no ha tenido contacto con contenidos académicos, ni praxis educativas inclusivas que los capacite para relacionarse con comunidades escolares diversas. Realidad que, necesariamente, los obliga a formarse, reflexionando no sólo acerca su forma de actuar sino también mostrándose dispuestos a asumir la condición de aprendices en los procesos de cambios que requiere la IE. Asimismo, se ha de tener presente que el desempeño docente debe responder a las demandas de la educación contemporánea, buscando asegurar el acceso y la permanencia en el sistema regular de todos/as los/as estudiantes, promoviendo en todo momento la universalización de las oportunidades. No obstante, para hacer ello posible, es necesaria una educación de calidad que permita al estudiantado prepararse, organizarse y aún adaptarse, pues, es en el espacio escolar donde los niños/as y jóvenes conviven e interactúan diariamente con todo tipo de compañeros/as. Y es en ese mismo entorno donde el profesorado interactúa y se hace parte de su cotidianeidad (Garnique, 2012).

La IE tiene, además, como una de sus premisas, la promoción de la transformación de la sociedad, y la participación activa del estudiantado en la escuela, para lo cual urge que el profesorado tenga conocimiento de las necesidades de todos/as sus estudiantes de modo que, puedan desarrollar acciones pedagógicas que incentiven el aprendizaje significativo en las distintas asignaturas (Caimi y Nunes da Luz, 2018). Lo anterior, atendiendo a que la escuela es un espacio de socialización en el que el estudiante busca construir conocimientos con la ayuda y subsidios del profesorado, para lo cual es imprescindible que durante la planificación de sus clases investiguen, reflexionen y traten de adaptar sus prácticas (Infante, 2010). Vale decir, el éxito de la IE depende tanto de conocimientos generales del profesorado acerca del tema, como del conocimiento específico de las características cognitivas, conductuales y sociales de sus estudiantes. Conocimiento que, le permitirá diseñar experiencias de aprendizaje y estrategias de enseñanza apropiadas para cada estudiante (Hastings \& Oakford, 2010). En otras palabras, la comprensión que posea el profesorado acerca de las características individuales de sus 
http://dx.doi.org/10.5902/1984686X67482

estudiantes y la implementación de estrategias de enseñanza apropiadas, son determinantes para el éxito de la IE en el aula.

De allí, que estudiar las cuestiones asociadas a la operacionalización de la escuela inclusiva, implica conocer tanto las concepciones del profesorado respecto de este paradigma de educación, como de las variables implicadas en dicho proceso. En este sentido, numerosas investigaciones revelan que son variadas las problemáticas que limitan o entorpecen la IE (Muñoz, López y Assael, 2015). Por lo pronto, las falencias en la formación inicial docente, la falta de apoyo de los directivos, la ausencia de trabajo en equipo con otros/as profesores/as, además de un elevado número de estudiantes por curso, son las restricciones más comunes. Sin contar que los estudiantes no son portadores de una única identidad, sino de varias otras que se intersecan, cruzan o superponen que, normalmente, terminan siendo invisibilizadas o ignoradas por la escuela (Guasp, Roselló y De la Iglesia, 2016).

Otra traba no menos relevante, es el sesgo compensatorio que aún prevalece en la política educativa nacional, que continúa poniendo el acento en el déficit o la carencia de ciertos grupos particulares (Matus, Rojas, Guerrero, Herraz y Sanyal, 2019; Iturra, 2019). Realidad que obliga a rediseñar las políticas de IE, no reduciéndola a la atención de las NEE o la discapacidad, sino abriéndola a otros temas y grupos excluidos.

\section{Método}

En lo metodológico, la investigación se encuadra en el enfoque cualitativo - descriptivo con un diseño de casos múltiples (Merrian, 1998; Stake, 1999), atendiendo a la multiplicidad de unidades de análisis, las cuales están conformadas por las universidades del CRUCH que imparten pedagogías. Desde el punto de vista de las técnicas de recolección de datos, se privilegió el análisis de documentos provenientes de internet (Bardín, 2002; Araneda, Parada y Vásquez, 2008), entendidos estos como "instrumento" de investigación; pues, como postula Fragoso, Recuero y Amaral (2011), internet puede ser objeto, lugar o instrumento de pesquisa.

De allí, que en este estudio internet sea abordada como un artefacto cultural, puesto que la red es entendida como un elemento de la cultura y no como una entidad aparte, caracterizada por la integración de los ámbitos on line y off line, donde se reconocen diferentes significados culturales en distintos contextos de uso. Es decir, el objeto de internet no es único, sino multifacetado y susceptible de múltiples apropiaciones (Fragoso, 
http://dx.doi.org/10.5902/1984686X67482

Recuero y Amaral 2011). Por otro lado, los niveles de privacidad de la documentación recolectada, pueden ser definida como pública, dado que está disponible y es de libre acceso a través de las páginas webs institucionales de las distintas universidades seleccionadas.

En lo que respecta a las unidades de análisis que orientaron la búsqueda y ayudaron a configurar el apartado de resultados, tenemos las siguientes: a) nociones relativas a IE; b) contenidos asociados a IE; c) competencias y/o habilidades asociadas a IE; y d) estrategias pedagógicas asociadas a IE.

Es importante consignar, asimismo que, tratándose de una muestra intencional, el corpus documental estuvo conformado, básicamente, por las mallas o itinerarios formativos de las distintas pedagogías, más los perfiles de egreso declarados en las respectivas páginas webs institucionales. Para una mejor comprensión del objeto de estudio y considerando el origen de las universidades, finalidades y, en algunos casos, confesionalidad de las mismas, de las 30 universidades que conforman el $\mathrm{CRUCH}$, se hizo una subdivisión entre aquellas denominadas universidades "públicas estatales", "publicas no estales" y "privadas".

En los siguientes cuadros se muestra la cantidad de pedagogías que imparte cada universidad dependiendo de su tipo de administración o dependencia.

Cuadro 1 - Número de pedagogías que imparten las universidades públicas estatales del CRUCH

\begin{tabular}{|c|c|c|}
\hline UNIVERSIDADES PÚBLICAS ESTATALES & $\begin{array}{l}\text { PEDAGOGÍAS } \\
\text { CASA CENTRAL }\end{array}$ & $\begin{array}{c}\text { PEDAGOGÍAS EM } \\
\text { SEDES }\end{array}$ \\
\hline U. de Chile & 4 & \\
\hline U. de Santiago de Chile & 5 & \\
\hline U. de Tarapacá & 7 & \\
\hline U. Arturo Prat & 6 & \\
\hline U. de Antofagasta & 7 & \\
\hline U. de Atacama & 4 & \\
\hline U. de La Serena & 10 & \\
\hline U. de Valparaíso & 5 & \\
\hline U. de Playa Ancha & 14 & 3 \\
\hline U. Metropolitana de C. de la Educación & 11 & \\
\hline \multicolumn{3}{|l|}{ U. Tecnológica Metropolitana ${ }^{3}$} \\
\hline U. de O'Higgins & 7 & \\
\hline U. de Talca & & 8 \\
\hline U. del Bío - Bío & & 8 \\
\hline U. de la Frontera & 6 & \\
\hline U. de Los Lagos & 8 & 4 \\
\hline \multicolumn{3}{|l|}{ U. de Aysen ${ }^{4}$} \\
\hline U. de Magallanes & 7 & \\
\hline Subtotal & 101 & 23 \\
\hline TOTAL & & \\
\hline
\end{tabular}

Fuente: Creación propia (2021). 
http://dx.doi.org/10.5902/1984686X67482

Cuadro 2 - Número de pedagogías que imparten universidades públicas no estatales del CRUCH

\begin{tabular}{|c|c|c|}
\hline UNIVERSIDADES PRIVADAS & $\begin{array}{l}\text { PEDAGOGÍAS } \\
\text { CASA CENTRAL }\end{array}$ & $\begin{array}{l}\text { PEDAGOGÍAS } \\
\text { SEDES }\end{array}$ \\
\hline PUC de Chile & 15 & 2 \\
\hline U. de Concepción & 14 & 5 \\
\hline \multicolumn{3}{|l|}{ U. Técnica Federico Santa María ${ }^{4}$} \\
\hline PUC de Valparaíso & 9 & \\
\hline U. Austral de Chile & 5 & 4 \\
\hline U. Católica del Norte & 3 & 1 \\
\hline \multicolumn{3}{|l|}{ U. Católica del Maule } \\
\hline U. Católica de la Santísima Concepción & 8 & \\
\hline U. Católica de Temuco & 11 & \\
\hline Subtotal & 65 & 12 \\
\hline TOTAL & \multicolumn{2}{|c|}{77} \\
\hline
\end{tabular}

Fuente: Creación propia (2021).

Cuadro 3 - Número de pedagogías que imparten universidades privadas del $\mathrm{CRUCH}$

\begin{tabular}{|l|c|c|}
\hline \multicolumn{1}{|c|}{ UNIVERSIDADES PRIVADAS } & $\begin{array}{c}\text { PEDAGOGÍAS } \\
\text { CASA CENTRAL }\end{array}$ & $\begin{array}{c}\text { PEDAGOGÍA } \\
\text { SEDES }\end{array}$ \\
\hline U. de Los Andes & 5 & \\
\hline U. Alberto Hurtado & 6 & \\
\hline U. Diego Portales & 6 & \\
\hline & & 17 \\
\hline
\end{tabular}

Fuente: Creación propia (2021).

En total, las universidades del CRUCH imparten 218 pedagogías distribuidas en los distintos niveles del sistema escolar. Considerando los posibles efectos distorsionadores que podría tener en los análisis, se excluyeron las pedagogías orientadas a la atención de niños/as y jóvenes con NEE como es Educación Especial y/o Diferencial. En este sentido cabe recordar que, en Chile, a diferencia de otros países de la región, existe formación específica de profesores/as a nivel de pregrado dedicada a asistir pedagógicamente a estos estudiantes.

Finalmente, para el análisis de la información proveniente de los documentos recolectados se recurrió al análisis de contenidos, dado que, como sostiene Bardín (2002), este permite ver la presencia o ausencia de una característica o de un conjunto de ellas presente en un determinado fragmento de mensaje. En otras palabras, a través de esta técnica es posible rastrear categorías sociales, culturales, educativas y metodológicas. Desde esta perspectiva, se puede descubrir, además, la filosofía que subyace o inspira un determinado proyecto o acción de una organización educativa, visualizando las contradicciones o coincidencias existentes (Araneda, Parada y Vásquez, 2008). 
http://dx.doi.org/10.5902/1984686X67482

\section{Resultados}

Analizada la oferta académica de las universidades del $\mathrm{CRUCH}$ que ofrecen pedagogías (27 de 30 universidades), se puede afirmar que, si bien la gran mayoría ha introducido en los últimos años asignaturas referidas a IE en los itinerarios formativos, en virtud de las exigencias ya comentadas del sistema escolar, de los nuevos estándares para la formación de profesores y de la CNA, ello no ocurre en todas las pedagogías y en todas las universidades con el mismo grado de celeridad. La explicación de esa desigual distribución y ritmos de las transformaciones se debe, probablemente, a la voluntad de las autoridades de las respectivas casas de estudio, a la capacidad de interpretar de esas mismas autoridades las exigencias de cambio, participación y democratización de las instituciones, a lo engorroso y burocrático que pueden resultar las innovaciones curriculares, particularmente en las universidades estatales que necesariamente requieren del cumplimiento de ciertas etapas y tiempos exigidos por ley, entre otros varios factores. En la práctica, han sido sólo las carreras innovadas, esto es, que han transitado de un currículo basado en objetivos a uno por competencias (u orientado por competencias), las que han introducido tales asignaturas.

En otras palabras, el tránsito ha sido gradual donde se ha producido, y ha beneficiado sólo a los estudiantes de los primeros años que han ingresado recientemente a las universidades. En general, los cursos más avanzados, o a punto de egresar, continúan con las mallas de formación antiguas (por objetivos), que no contemplaban asignaturas de IE o de género, que es otra de las reivindicaciones, levantadas por el movimiento feminista durante las movilizaciones universitarias de 2018 que paralizaron prácticamente a la mayor parte de las universidades del país, que, entre otras cosas, exigían una educación no sexista que alcanzara, transversalmente, a toda la formación de profesionales en Chile.

En los siguientes cuadros, se muestran la cantidad de asignaturas referidas a IE introducidas por las distintas universidades. Con todo, como ya se comentó, no en todas las pedagogías que imparte una misma institución, se han incorporado, ni tampoco en todos los niveles de una misma carrera, focalizándose más bien en los primeros años. 
http://dx.doi.org/10.5902/1984686X67482

Cuadro 4 - Consolidado de asignaturas referidas a inclusión educativa en universidades públicas estatales del $\mathrm{CRUCH}$

\begin{tabular}{|l|c|}
\hline UNIVERSIDADES PÚBLICAS ESTATALES & $\begin{array}{c}\text { ASIGNATURAS REFERIDAS } \\
\text { A INCLUSIÓN }\end{array}$ \\
\hline U. de Chile & 4 \\
\hline U. de Santiago de Chile & 4 \\
\hline U. de Tarapacá & 7 \\
\hline U. Arturo Prat & 2 \\
\hline U. de Antofagasta & 6 \\
\hline U. de Atacama & 1 \\
\hline U. de La Serena & \\
\hline U. de Valparaíso & 2 \\
\hline U.de Playa Ancha & 11 \\
\hline U. Metropolitana de C. de la Educación & 6 \\
\hline U. Tecnológica Metropolitana & 10 \\
\hline U.de O'Higgins & 7 \\
\hline U.de Talca & 8 \\
\hline U. del Bío - Bío & 1 \\
\hline U. de la Frontera & \\
\hline U. de Los Lagos & 7 \\
\hline U. de Aysen & 76 \\
\hline U. de Magallanes & \\
\hline
\end{tabular}

Fuente: Creación propia (2021).

Como se puede observar, en el cuadro 4 aparece el número de asignaturas referidas a IE que imparten las distintas pedagogías de las universidades publicas estatales.

Cuadro 5 - Consolidado de asignaturas referidas a inclusión educativa en universidades públicas no estatales del $\mathrm{CRUCH}$

\begin{tabular}{|l|c|}
\hline \multicolumn{1}{|c|}{ UNIVERSIDADES PÚBLICAS NO ESTATALES } & $\begin{array}{c}\text { ASIGNATURAS REFERIDAS } \\
\text { A INCLUSIÓN }\end{array}$ \\
\hline PUC de Chile & 11 \\
\hline U. de Concepción & 12 \\
\hline U. Técnica Federico Santa María & 7 \\
\hline PUC de Valparaíso & 8 \\
\hline U. Austral de Chile & 7 \\
\hline U. Católica del Norte & 7 \\
\hline U. Católica del Maule & 7 \\
\hline U. Católica de la Santísima Concepción & \\
\hline U. Católica de Temuco & $\mathbf{5 9}$ \\
\hline
\end{tabular}

Fuente: Creación propia (2021).

En el cuadro anterior, aparece la distribución de asignaturas de IE impartidas por las universidades publicas no estatales, que si bien menos en términos absolutos si comparadas con las instituciones publicas estatales resultan más significativas porque están repartidas solo en 7 instituciones a diferencia de las 14 estatales. 
http://dx.doi.org/10.5902/1984686X67482

Cuadro 6 - Consolidado de asignaturas referidas a inclusión educativa en universidades privadas del $\mathrm{CRUCH}$

\begin{tabular}{|c|c|}
\hline UNIVERSIDADES PÚBLICAS NO ESTATALES & $\begin{array}{c}\text { ASIGNATURAS REFERIDAS } \\
\text { A INCLUSIÓN }\end{array}$ \\
\hline U. de Los Andes & 3 \\
\hline U. Alberto Hurtado & 5 \\
\hline U. Diego Portales & 5 \\
\hline TOTAL & 13 \\
\hline
\end{tabular}

Fuente: Creación propia (2021).

De los cuadros anteriores se desprende que, la gran mayoría de las universidades del $\mathrm{CRUCH}$, no importando sus dependencias, aparatos burocrático - administrativos o fines, han hecho esfuerzos por responder a los requerimientos de sociales y legales de inclusión, no evidenciándose diferencias sustantivas en sus motivaciones o deseos de satisfacer dichos requerimientos. Las diferencias, como veremos más adelante, afloran por los acentos, ciertos contenidos y omisiones generadas por las implicancias de algunas perspectivas de género o pensar la inclusión de ciertos grupos disidentes que ponen en tela de juicio o cuestionan algunas doctrinas de corte religioso que inspiran a ciertas universidades confesionales.

\section{Homologación de la IE con las NEE}

Un primer aspecto que llama la atención, es que la casi totalidad de universidades que han asumido el desafío de incorporar la IE en los itinerarios formativos de las distintas pedagogías, lo han hecho homologando inclusión con la atención de estudiantes con NEE. Con todo, tal preocupación resulta sesgada aun dentro del ámbito de las NEE, pues, privilegia exclusivamente la atención de estudiantes con problemas de aprendizaje o descendidos académicamente, despreocupándose totalmente de aquellos considerados talentosos o superdotados, que son otra forma de NEE, pero en sentido inverso. No obstante, tal descuido no es sólo de las universidades, sino del propio Ministerio de Educación (MINEDUC) y sus políticas educativas que nada dicen en relación a estos últimos estudiantes. Tal es así, que los escasos programas de atención de estudiantes talentosos en el país, son producto de iniciativas privadas de algunas universidades, en su mayoría públicas no estatales y/o confesionales, y no de la política pública.

Otra debilidad detectada en los esfuerzos de plasmar la IE en los itinerarios formativos es, pese a las críticas de los propios "formadores de formadores, el marcado acento medicalizador y normativo que prevalece en muchas de esas asignaturas (CORNEJO, 2019a). En este sentido, gran preocupación se evidencia por los diagnósticos de los 
http://dx.doi.org/10.5902/1984686X67482

distintos trastornos de aprendizaje y sus respectivos tratamientos, por la normativa que distingue entre NEE transitorias y permanentes, o las subvenciones (subsidios económicos adicionales del Estado) a las escuelas regulares que reciben este tipo estudiantes, con lo cual se sigue reforzando el paradigma medicalizador que aún prevalece en el sistema educativo chileno (GARCÍA y LÓPEZ, 2019; ITURRA, 2019). Es más, no son pocas, las casas de estudio que, además, de introducir asignaturas específicas alusivas a NEE, han reforzado las prácticas profesionales en esta misma línea.

Ello no tendrían ningún reparo, si asociada a esa preocupación se promoviese, igualmente, una visión más amplia de la inclusión de modo de pensar en otros grupos excluidos presentes en los sistemas educativos formales, o temáticas que demandan similar atención en las escuelas, entre ellas, la promoción y defensa de los derechos humanos de esos mismos niños/as y jóvenes que demandan ser incluidos, el reconocimiento de su ciudadanía plena a fin de terminar con las nociones medicalizadas y/o "paternalistas" que suelen prevalecer en relación a todos los/as estudiante excluidos, entre otros varias asuntos.

Sin embargo, no se puede desconocer que una cuota importante de esta prevalencia de las NEE y de la matriz medicalizadora, proviene de los propios profesores del sistema escolar que, normalmente, suelen asociar la inclusión con NEE. No son pocos los que, consultados por la IE, mecánicamente, más allá del grupo específico por el cual se les esté consultando, comiencen a hablar de NEE (OJEDA, 2019; ARAVENA, 2021). Tal asociación, sin duda, ha hecho que las universidades seleccionadas a la hora de pensar en inclusión privilegien esta perspectiva por sobre otras; realidad particularmente perceptible en universidades más preocupadas con el cumplimiento de la norma (estándares pedagógicos, acreditación, etc.), que con los desafíos e interpelaciones que implica la IE o la filosofía y subsecuente convivencia cotidiana con la diversidad.

En este mismo sentido, no se pueden obviar las complicaciones y aprietos que supone la inclusión para las universidades confesionales, no sólo para abordar ciertos temas e incluir a ciertos grupos, tradicionalmente excluidos en virtud de sus afecciones erótico afectivas y/o identidades o expresiones de género, sino también por las exigencias democratizadoras, de reconocimiento y participación de sus propias comunidades universitarias. De allí, que junto con las distintas demandas sociales que tienden a la homologación, no se pueda desconocer lo cómodo y conveniente que resulta para estas 
instituciones la asociación entre NEE e IE; pues, así se cumple con la ley, al mismo tiempo que nadie puede cuestionar sus esfuerzos inclusivos.

La excepción a esta tendencia, focalizada en la atención de las NEE, la conforman algunas universidades que junto con ampliar la reflexión acerca de la IE, se han embarcado en intensas campañas de sensibilización de la comunidad universitaria, donde las asignaturas de inclusión resultan ser una pieza más dentro de ese proceso reflexivo y democratizador. Igualmente, alentador, son algunas especializaciones o menciones referidas a inclusión que han ido surgiendo en carreras como Pedagogía en Filosofía, de la Universidad de Ciencias de la Educación (UMCE).

Con todo, sin desconocer los avances antes enunciados, del análisis general de las universidades seleccionadas y de las asignaturas implementadas, se puede afirmar, que más allá del tipo de universidad de que se trate, y de las razones que fundamenten la perspectiva escogida, sigue prevaleciendo la asociación NEE e IE.

\section{La tímida irrupción de la interculturalidad}

Pese a la multiplicación de discursos oficiales y de ciertos sectores de la sociedad civil que hablan de un Chile multicultural, que aspira al reconocimiento constitucional de sus pueblos originarios, que elucubra con la riqueza de sus migrantes y la reconfiguración racial, en la práctica son escasas las iniciativas políticas y académicas que se hacen cargo de esa diversidad. Más sorprendente aún es constatar que en los itinerarios formativos de las distintas pedagogías son escasas las alusiones a la interculturalidad, focalizándose más bien en aspectos teóricos generales que en estrategias específicas de trabajo pedagógico para desarrollar en el aula.

La excepción a esa tendencia y descuido la constituye la U. Católica de Temuco (UCT). Universidad localizada en el corazón del territorio indígena del país, que ha transversalizado la interculturalidad a todas las pedagogías (aun cuando concentrada en el estudio e intervenciones en cultura y contextos mapuches), ofreciendo 34 asignaturas y electivos, superando con mucho la oferta en torno a las NEE que ofrecen el resto de las universidades nacionales que, en general, por carrera no superan las 2 asignaturas en el mejor de los casos. A lo anterior, en el caso de la UCT se suma, además, una pedagogía especializada en interculturalidad indígena (Pedagogía en educación básica intercultural en contexto mapuche). Experiencia inédita, quizás homologable a la educación diferencial que se ofrece en el ámbito de las NEE. 
http://dx.doi.org/10.5902/1984686X67482

La otra experiencia que podría aproximarse, aun cuando de mucho menor envergadura y más orientada a la sensibilización que a la propuesta por la UCT, es la desarrollada por la U. Arturo Prat, que desde una perspectiva más amplia, ya no centrada exclusivamente en la cuestión indígena, intenta problematizar el tema de la interculturalidad tanto en su vertiente indigenista cuanto migratoria a través de una asignatura llamada: "Interculturalidad, pueblos originarios, realidad nacional y transfronteriza" que imparte en todas sus pedagogías.

No obstante, estas iniciativas, no deja de llamar la atención el descuido por la temática, tanto de aquellas universidades localizadas en territorio indígena (norte grande, Araucanía, Los Ríos y Los Lagos), cuanto de aquellas otras situadas en zonas (principalmente urbanas) con alta concentración de migrantes (norte, Gran Santiago, y Valparaíso). Población que en la actualidad representan más o menos el 10\% de la población total del país (Departamento de Extranjería y Migración, 2021).

\section{Migrantes en escena}

Estrechamente relacionado con la interculturalidad, aparece la IE de los estudiantes migrantes. Realidad que se ha incrementado significativamente en la última década, no sólo por la multiplicación de países de procedencia, sino también por la diversificación de las edades de los migrantes. Si en las décadas pasadas la migración era preferentemente de personas jóvenes o adultos jóvenes en edad laboral, en los últimos años esa matriz se ha diversificado.

De acuerdo a antecedentes aportados por el Servicio Jesuita de Migrantes (2020), la matrícula escolar en Chile entre 2014 y 2019 ha aumentado un 616\%, y casi se ha triplicado desde 2016. En lo que respecta al tipo de establecimientos a los que asisten estos estudiantes, el informe señala que más de la mitad lo hace a escuelas públicas o municipalizadas, y sólo un tercio a escuelas subvencionadas. De igual modo, se constata que el estudiantado nacional ha comenzado a "migrar" de las escuelas públicas a las subvencionadas en búsqueda de mejores oportunidades. Por otra parte, no se puede desconocer que la presencia de estudiantes migrantes en establecimientos públicos ha ayudado a atenuar el descenso de matrícula en este tipo de establecimientos entre 2014 y 2019, contribuyendo a un leve aumento después de 2016

En cuanto a la distribución geográfica para 2019, el mismo informe señala que el $78 \%$ de los estudiantes migrantes se concentraba en cuatro regiones del país: Arica y 
Parinacota, Tarapacá, Antofagasta y Región Metropolitana, en gran parte debido a las oportunidades laborales que ofrecen y/o la cercanía de las zonas fronterizas (SERVICIO JESUITA DE MIGRANTES, 2020)5. Es importante subrayar que todas estas cifras son provisorias, dado que los flujos migratorios informales no han cesado pese al cierre de fronteras motivado por la pandemia.

Más allá de las cifras, lo emergente del tema y de las oportunidades de estudio que ofrece la nueva configuración del sistema educacional chileno, se verifica que la totalidad de las universidades del $\mathrm{CRUCH}$ no presentan asignaturas específicas en los itinerarios formativos de profesores que se hagan cargo de esa nueva realidad escolar 6 , particularmente en las escuelas públicas donde tienden a concentrarse este tipo de estudiantes.

Las escasas alusiones a la migración, se inscriben en reflexiones teóricas genéricas, insertas en algunas asignaturas que abordan la diversidad, la tolerancia, los derechos humanos o la interculturalidad de modo superficial, pero en ningún caso aportan pistas específicas para el trabajo en el aula o proporcionan metodologías para la inclusión efectiva de los estudiantes migrantes en las distintas comunidades escolares. Situación que poco ayuda a romper con las tendencias asimilacionistas y homogeneizadoras que aún prevalecen en las escuelas nacionales.

De hecho, junto con la queja reiterativa del profesorado de las distintas escuelas del país de no contar con las competencias necesarias para trabajar con estudiantes con NEE, debido a que no habrían sido preparados por sus respectivas universidades, aparece una queja similar en relación a los estudiantes migrantes (ARAVENA, 2021; OJEDA, 2019; CORNEJO, 2019a). En la práctica, como mencionan esos mismos profesores del sistema escolar, los aciertos en el trabajo con migrantes han sido, básicamente, producto de la convivencia cotidiana de esos mismos docentes con estos niños/as y jóvenes, y no producto de capacitaciones o formaciones sistemáticas, menos aún de recursos adicionales allegados a las escuelas con estos fines. En ese sentido, la política nacional de atención a estudiantes migrantes divulgada por el MINEDUC, contenida en una serie de orientaciones presentes en la web institucional y folletería alusiva al tema, no ha asumido directamente la capacitación del profesorado en ejercicio. Tampoco, a diferencia de lo que ocurre con las NEE, se cuenta con profesionales de apoyo o recursos adicionales en las escuelas para la atención de estos/as estudiantes. 
http://dx.doi.org/10.5902/1984686X67482

De allí, que las omisiones de las universidades en esta temática, más allá de las arengas políticas, no resulten tan extrañas a la luz de los antecedentes anteriores, toda vez que la migración no aparece como tópico de reflexión, ni mucho menos como praxis educativa a nivel de pregrado. Las ofertas de formación sistemática para el trabajo específico con migrantes, han comenzado a irrumpir, más bien, en cursos de especialización o de postgrados de algunas universidades como, por ejemplo, el diplomado en Migración, políticas públicas y estrategias de inclusión de la U. Tecnológica Metropolitana (UTEM) o el diplomado en Migraciones, integración y diversidad cultural de la U. Alberto Hurtado, por nombrar sólo algunos.

Otras de las formas como ha comenzado a instalarse el tema, es a través de líneas de investigación en algunos programas de postgrado relacionados con las humanidades y/o ciencias sociales como, por ejemplo, el Doctorado en Educación de la U. de Tarapacá (UTA), que en su línea de educación y cultura apuesta por el estudio de los fenómenos relacionados con educación y cultura en espacios transfronterizos donde tienen lugar las migraciones (UTA, 2021), o el doctorado de Ciencias Sociales de esa misma casa de estudio, que tiene entre sus líneas: Movilidad, migraciones y frontera.

Recapitulando, podemos decir entonces, que la preparación de los futuros profesores en materia de inclusión de migrantes, es aún una tarea pendiente tanto porque la inclusión de los estudiantes con NEE concentra la atención de los planes de estudio de la mayor parte de las pedagogías que se imparten en el país, cuanto por el desinterés de las propias casas de estudio de formar profesores en interculturalidad con las competencias necesarias para atender a estudiantes culturalmente diversos, como pueden ser los migrantes o los indígenas. Con todo, el desinterés a que hacíamos mención, no se debe apenas a la excesiva focalización en las NEE, sino también a la falta de preparación de los propios "formadores de formadores" (CORNEJO, 2019a), pues, como bien se señala en el Marco para la buena enseñanza: "nadie puede enseñar lo que no sabe" (CPEIP, 2008, p. 16).

\section{Género omitido, disidencias silenciadas}

Si indígenas y migrantes aparecen tímidamente en los itinerarios formativos de las universidades del $\mathrm{CRUCH}$, mucho más reciente y escuálida es la presencia de temáticas de género, las reivindicaciones feministas y el reconocimiento de las disidencias sexuales y de género. Todas ellas producto de las presiones y movilizaciones estudiantiles, que a procesos reflexivos o de maduración de las distintas comunidades universitarias del país. 
http://dx.doi.org/10.5902/1984686X67482

De hecho, los incipientes avances en estas materias han sido producto de las movilizaciones feminista que se comenzaron a gestar a partir de mayo de 2018, entre cuyas reivindicaciones y exigencias aparecían: protocolos de denuncia del acoso y/o abuso sexual, violencia de género, discriminación arbitraria, paridad entre hombres y mujeres en el acceso a los más altos cargos directivos y de gestión de las universidades, término de la educación sexista y reconocimiento de las disidencias (CORNEJO, 2021).

En este sentido, los mayores avances en estas materias se evidenciaron en las universidades laicas, públicas estatales y públicas no estatales del CRUCH. Por el contrario, las universidades confesionales no sólo están al debe en estos asuntos, sino que se han mostrado muy reticentes a incorporar estos tópicos en los planes de estudio de las distintas pedagogías (o de cualquier carrera), especialmente cuando ellos aluden a los derechos sexuales y reproductivos, al carácter performático del género (BUTLER, 2006; BUTLER, 2007; BUTLER, 2009), a la igualdad efectiva entre hombres y mujeres, particularmente en el acceso a los cargos directivos, o al reconocimiento de las disidencias; promoviendo más bien para estos últimos, la "compasión" en lugar del reconocimiento de sus derechos (CORNEJO, 2019b).

Una de las acciones más claras que evidencia la voluntad de las universidades laicas de asumir los cuestionamientos del movimiento feminista universitario, fue la creación de unidades o direcciones de género, claramente identificables en los respectivos organigramas institucionales y cuya finalidad es la sensibilización y formación de las comunidades universitarias; además de cumplir funciones de acogida y seguimiento de las denuncias de acoso y/o abuso sexual. En las universidades confesionales, en cambio, estas unidades son inexistentes o con atribuciones muy limitadas, lo que deja al descubierto el carácter nominal de las mismas. En los que respecta a los protocolos de denuncia del acoso y/o abuso sexual, violencia de género y discriminación arbitraria, ellos están presentes en la casi totalidad de las universidades del $\mathrm{CRUCH}$, salvo algunas universidades confesionales, que nuevamente dejan entrever sus desconfianzas respecto de estos dispositivos de control de las políticas de género a nivel nacional.

Donde sí se asemejan la mayor parte de las universidades laicas y confesionales es en el incumplimiento del compromiso asumido, en el contexto de las movilizaciones feministas de 2018, de terminar con la educación sexista que aún prevalece en la formación de profesionales en Chile. De una treintena de casas de estudio que conforman el $\mathrm{CRUCH}$, sólo 5 de ellas (todas instituciones laicas) han incorporado alguna asignatura referida a 
género en los itinerarios formativos de profesores/as. Haciendo una revisión de norte a sur del país tenemos: la U. Arturo Prat que incorporó 1 asignatura en Educación Parvularia; la U. de La Serena que incorporó 1 asignatura en todas las pedagogías que imparte (en total 11), constituyéndose en la institución más destacada en este punto a nivel nacional; la $U$. de Valparaíso con 1 asignatura en 2 de sus pedagogías; la U. Metropolitana de Ciencias de la Educación (UMCE) con 3 asignaturas de especialización en Pedagogía en Filosofía; y la U. del Bío - Bío con 4 asignaturas en 4 de las 8 pedagogías que imparte (CORNEJO, 2021). La excusa recurrente por este descuido ha sido el estallido social de finales de 2019 y la posteriormente pandemia. Con todo, tal excusa se debería ser relativizada, pues, pese a esos acontecimientos los procesos de innovación de las distintas carreras han continuado.

En lo que respecta a las disidencias los avances más significativos se han dado en las universidades laicas, que no sólo han acogido la demanda de reconocimiento del nombre social de los estudiantes trans al interior de las mismas, aun cuando no se haya concretado el cambio del nombre registral o legal $^{7}$, sino que cuentan, además, con protocolos de denuncia de la discriminación homolesbotransfobica.

En el ámbito curricular, la situación es mucho más desalentadora, pues, salvo menciones muy superficiales en las pocas asignaturas específicas de género incorporadas en las mallas de formación de profesores, la temática está completamente ausente o, mejor aún, silenciada en virtud de la falta de convicción de que los disidentes sean, realmente, sujetos de derechos. Para muchos se trataría más bien de opciones o preferencias sexuales, motivo por el cual no cabrían dentro de la IE.

\section{Conclusiones}

A modo de conclusión, cabría decir que la IE, en el contexto de la formación de profesores en las universidades de $\mathrm{CRUCH}$, es de "sombras y luces", pues, son indudables los avances si comparado con lo que ocurría apenas a inicio de la presente década, donde el tema era considerado "tópico de especialistas" y frente al cual los/as profesores/as, en general, sentían que no les correspondía pronunciarse, ni menos aún formarse. Tal percepción comenzó, paulatinamente, a cambiar a medida que las exigencias del sistema escolar se multiplicaron, demandando de mejores y más preparados profesores/as que interactuasen con comunidades escolares cada vez más diversas en virtud de la creciente incorporación de estudiantes con NEE a las escuelas regulares, la multiplicación del estudiantado migrante y los debates en torno al necesario tránsito de una escuela 
http://dx.doi.org/10.5902/1984686X67482

monocultural a una intercultural, con el consecuente reconocimiento de la multiplicidad de grupos presentes hoy en las escuelas chilenas.

Y si bien las universidades del $\mathrm{CRUCH}$, asumieron el desafío formativo, unas veces por convicción y otras por las presiones sociales y legales del medio, no se puede desconocer que dicho proceso no ha sido regular o con la misma intensidad y profundidad en todas las casas de estudio, tendiéndose a centrar la innovación curricular y subsecuente formación de profesores en la atención de los/as estudiantes con NEE, con descuido de otros grupos (pese a representar una porción significativa del estudiantado nacional, especialmente en las escuelas públicas), o el abordaje de temáticas que subvierten el orden patriarcal - heterosexista.

En esta última dimensión, particularmente cuestionable, ha sido la actitud de las universidades confesionales, que si bien han destacado en la formación de profesores/as en NEE y en algunos casos en interculturalidad, por razones ideológico-religiosas, se han resistido a debatir y mucho más incorporar en los itinerarios formativos temáticas referidas a género o de reconocimiento de las disidencias sexuales y/o de género, lo cual no sólo representa un sesgo, sino una traba a los procesos de inclusión efectiva.

De allí, que los mayores desafíos del sistema educativo nacional y de las universidades en particular, en tanto entidades formadoras, sean tanto ampliar las nociones y grupos beneficiarios de la IE, cuanto empaparse de la filosofía que subyace a la misma que no admite exclusiones de ningún tipo, cualquiera sean las razones esgrimidas.

\section{Referências}

ARANEDA, Aladino; PARADA, María Verónica; VÁSQUEZ, Alicia. Investigación cualitativa en educación y pedagogía. Concepción: Editorial UCSC, 2008.

ARAVENA, Daniel. Problemas y Desafíos ante la inclusión educativa en la formación de profesores de cuatro universidades del centro - sur de Chile. Talca: Universidad Católica del Maule, 2021. 1 CD-ROM.

BARDÍN, Laurence. Análisis de contenido. Madrid: Ediciones Akal, 2002.

BUTLER, Judith. Performatividad, precariedad y políticas sexuales. AIBR. Revista de Antropología Iberoamericana, v. 4, n. 3, p. 321-336, 2009.

BUTLER, Judith. Deshacer el género. Buenos Aires: Paidós, 2006.

BUTLER, Judith. El género en disputa. El feminismo y la subversión de la identidad. Barcelona, Paidós, 2007. 
CAIMI, Flavia; NUNES DA LUZ, Rosangela. Inclusão no contexto escolar: Estado do conhecimento. Revista Educação Especial, v. 31, n. 62, p. 665 - 668, mar 2018.

CHILE. Centro de Perfeccionamiento, Experimentación e Investigaciones Pedagógicas (CPEIP). Marco para la buena enseñanza. Santiago: CPEIP / MINEDUC, 2008. Disponible en: https://www.cpeip.cl/wp-content/uploads/2019/01/Marco-buenaensenanza.pdf. Acceso en: 15 ago. 2021.

CHILE. Centro de Perfeccionamiento, Experimentación e Investigaciones Pedagógicas (CPEIP). Estándares orientadores para la formación inicial docente. Santiago: CPEIP /MINEDUC, 2016. Disponible en: https://www.cpeip.cl/estandares-formacion-docente/. Acceso en: 10 jul. 2021.

CHILE. Ministerio de Educación (MINEDUC). Política nacional de estudiantes extranjeros: 2018 - 2022. Santiago: MINEDUC, 2018. Disponible en:

https://migrantes.mineduc.cl/wp-content/uploads/sites/88/2020/04/Pol\%C3\%ADticaNacional-Estud-Extranjeros.pdf. Acceso en: 10 jul. 2021.

CHILE. Comisión Nacional de Acreditación (CNA). Criterios y estándares para carreras de pedagogía en Chile. Santiago: CNA, 2019. Disponible en:

https://investigacion.cnachile.cl/archivos/cna/documentos/Propuesta-Criterios-y-

Estandares-Pedagogaia-FINAL.pdf. Acceso en: 10 jul. 2021.

CHILE. Departamento de Extranjería y Migraciones. Estadísticas migratorias. Santiago: DEM, 2020. Disponible en: https://www.extranjeria.gob.cl/estadisticas-migratorias/. Acceso en: 10 jul. 2021.

CORNEJO, Juan Gender in the contexto of teacher training in Chile. Turkish Online Journal Qualitative Inquiry, v.12, n. 4, p. 324 - 332, jun 2021.

CORNEJO, Juan. Inclusión educativa desde la óptica de los académicos responsables de la formación de profesores de la Universidad Católica del Maule - Chile. Revista de Educación, v. XXVIII, n. 55, p. 27-58, sept 2019a.

CORNEJO, J. Nuevos excluidos en el sistema educacional chileno: problemas y desafíos. Revista Páginas de Educación, v. 12, n. 1, p. 28 - 48, feb 2019b.

FRAGOSO, Suely; RECUERO, Raquel; AMARAL, Adriana. Métodos de pesquisa para internet. São Paulo: Editora Sulina, 2011.

GARCÍA, Rosalba. y LÓPEZ, Verónica. Políticas de educación especial en Chile (2005 2015): Continuidades y cambios. Revista Brasileira de Educação Especial, v. 25, n. 1, p. 1-16, jan- mar 2019.

GARNIQUE, Felicita. Las representaciones sociales. Los docentes de educación básica frente a la inclusión escolar. Perfiles Educativos, v. XXXIV, n. 137, p. 99 - 118, sept 2012.

GUASP, Joan; ROSELLÓ, María Rosa; DE LA IGLESIA, Begoña. Buenas prácticas en educación inclusiva. Educatio Siglo XXI, v. 34, n. 1, p. 31 - 50, mar 2016. 
http://dx.doi.org/10.5902/1984686X67482

HASTINGS, Richard; OAKFORD, Suzanne. Student Teachers' Attitudes Towards the Inclusion of Children with Special Needs. Educational Psychology, v. 23, n. 1, p. 87 - 94 , jul 2010.

INFANTE, Marta. Desafíos a la formación docente: Inclusión educativa. Estudios Pedagógicos, v. XXXVI, n. 1, p. 287 - 297, jul 2010.

ITURRA, Pamela. Dilemas de la inclusión educativa en el Chile actual. Revista Educación las Américas, n. 8, p. 95 - 111, ene - jul 2019.

OJEDA, Grawen. Problemas y desafíos para la inclusión de género de niñas y niños migrantes que asisten a escuelas básicas de distinta dependencia de la región del Maule. Talca: Universidad Católica del Maule, 2019. 1 CD-ROM.

PAINEO, Jocelyn. Antecedentes históricos de la educación especial en Chile. Santiago: COANIL, 2012. Disponible en: https://es.slideshare.net/JocelynPaineo1/historiade-la-educacion-especial-15487382. Acceso en: 10 jul. 2021.

MATUS, Claudia; ROJAS, Carolina; GUERRERO, Patricia; HERRAZ, Pablo; SANYAL, Anita. Diferencia y normalidad: producción etnográfica e intervención en escuelas. Magis, v. 11, n. 23, p. 23 - 38, ene 2019.

MERRIAM, Sharan. Qualitative research and case study applications in education. San Francisco: Jossey-Bass Publishers, 1998.

MUÑOZ, María Loreto; LÓPEZ, Mauricio; ASSAEL, Jenny. Concepciones docentes para responder a la diversidad: ¿barreras o recursos para la inclusión educativa?

Psicoperspectivas, v. 14, n. 3, p. 68 - 79, sept 2015.

SERVICIO JESUITA MIGRANTE. Acceso e inclusión de personas migrantes en el ámbito educativo. Informe 2. Santiago: Servicio Jesuita, 2020. Disponible en: https://www.migracionenchile.cl/wp-content/uploads/2020/06/Informe-2Educaci\%C3\%B3n-2020.pdf. Acceso en: 15 ago. 2021.

STAKE, Robert. Investigación con estudio de casos. Madrid: Ediciones Morata, 1999.

VALENZUELA, Jorge; MUÑOZ, Carla; MARFULLJENSEN, Marisol. Perfiles motivacionales durante la formación inicial docente. Revista de currículo y formación del profesorado, v. 22, n. 1, p. 325 - 346, mar 2018.

\section{Notas}

${ }^{1}$ El Consejo de Rectores de las Universidades Chilenas (CRUCH) es un organismo colegiado, autónomo, con personalidad jurídica de derecho público, que tiene por función la coordinación del quehacer de las instituciones que lo conforman, para procurar un mejor rendimiento y calidad de la enseñanza superior del país. Fue creado por la Ley 11.575 /1954, y dotado de personalidad jurídica en 1964. Su Estatuto Orgánico actual está contenido en el Decreto con Fuerza de Ley № 2 de 1985 del Ministerio de Educación (MINEDUC). Forman parte de este organismo 27 rectores de las universidades estatales y no estatales públicas creadas o reconocidas con anterioridad a 1981 o que se derivan de aquellas, y de las creadas por ley. En 2018, por primera vez, se integraron 2 universidades privadas: Universidad Alberto Hurtado y Universidad Diego Portales, a las que se sumó la Universidad de Los Andes en 2019. 
2 En Chile hay un total de 56 universidades, de las cuales 30 hacen parte del $\mathrm{CRUCH}$, las que a su vez se subdividen en universidades públicas estatales (18), universidades públicas no estatales (9) y universidades privadas recientemente incorporadas (3).

${ }^{3}$ Tanto la U. Tecnológica Metropolitana (UTEM), cuanto la U. Técnica Federico Santa María, pese a ser consideradas universidades tradicionales, dado el carácter técnico de las mismas, no imparten pedagogías.

${ }^{4}$ La U. de Aysen de reciente creación (2015), tampoco ofrece pedagogía en ninguno de los niveles de enseñanza escolar.

${ }^{5}$ Para mayores detalles acerca de la política nacional de educación para la atención de estudiantes migrantes, consultar: MINEDUC (2018). Política nacional de estudiantes extranjeros: 2018 - 2022.

${ }^{6}$ En la actualidad, prácticamente en todas las regiones del país, aun las zonas más rurales o alejadas de la capital o de los territorios transfronterizos por donde suelen circular los migrantes, es posible encontrar estudiantes migrantes. Y si bien su presencia no es masiva como en estos últimos territorios, no dejan de representar un desafío de inclusión para las comunidades escolares locales.

${ }^{7}$ El Estado de Chile se ha comprometido a aplicar los Principios de Yogyakarta que, en el $\mathrm{N}^{\circ} 17$ de dicho documento establece: "Todas las personas tienen el derecho al disfrute del más alto nivel posible de salud física y mental, sin discriminación por motivos de orientación sexual o identidad de género". Igual compromiso ha asumido con lo indicado en la Ley 20.609/2012 Antidiscriminación que, en su artículo $3^{\circ}$ señala: "Toda persona tiene derecho a ser reconocida e identificada conforme a su identidad de género, una vez realizada la rectificación que regula esta Ley". En esta misma norma se subraya que no será condición para el reconocimiento del derecho a la identidad de género haberse sometido a algún tipo de intervención o tratamiento modificatorio de la apariencia. 